\title{
Metabolic remodeling during somatic cell reprogramming to induced pluripotent stem cells: involvement of hypoxia- inducible factor 1
}

\author{
Tomoaki Ishida ${ }^{1,2}$, Shu Nakao ${ }^{1,2}$, Tomoe Ueyama ${ }^{1,2}$, Yukihiro Harada ${ }^{1,2}$ and Teruhisa Kawamura ${ }^{1,2^{*}}$
}

\begin{abstract}
Induced pluripotent stem cells (iPSCs) were first established from differentiated somatic cells by gene introduction of key transcription factors, OCT4, SOX2, KLF4, and c-MYC, over a decade ago. Although iPSCs can be applicable for regenerative medicine, disease modeling and drug screening, several issues associated with the utilization of iPSCs such as low reprogramming efficiency and the risk of tumorigenesis, still need to be resolved. In addition, the molecular mechanisms involved in the somatic cell reprogramming to pluripotency are yet to be elucidated. Compared with their somatic counterparts, pluripotent stem cells, including embryonic stem cells and iPSCs, exhibit a high rate of glycolysis akin to aerobic glycolysis in cancer cells. This is known as the Warburg effect and is essential for maintaining stem cell properties. This unique glycolytic metabolism in iPSCs can provide energy and drive the pentose phosphate pathway, which is the preferred pathway for rapid cell proliferation. During reprogramming, somatic cells undergo a metabolic shift from oxidative phosphorylation (OXPHOS) to glycolysis trigged by a transient OXPHOS burst, resulting in the initiation and progression of reprogramming to iPSCs.

Metabolic intermediates and mitochondrial functions are also involved in the epigenetic modification necessary for the process of iPSC reprogramming. Among the key regulatory molecules that have been reported to be involved in metabolic shift so far, hypoxia-inducible factor 1 (HIF1) controls the transcription of many target genes to initiate metabolic changes in the early stage and maintains glycolytic metabolism in the later phase of reprogramming. This review summarizes the current understanding of the unique metabolism of pluripotent stem cells and the metabolic shift during reprogramming, and details the relevance of HIF1 in the metabolic shift.
\end{abstract}

Keywords: Induced pluripotent stem cells, Reprogramming, Metabolic shift, Hypoxia-inducible factor, Regenerative medicine, Glycolysis, Oxidative phosphorylation

\section{Background}

Induced pluripotent stem cells (iPSCs) are established by the introduction of reprogramming factors (OCT4, SOX2, KLF4, and c-MYC) into somatic cells. Over a decade ago, Takahashi and colleagues reported that iPSCs can be generated from mouse and human somatic

\footnotetext{
* Correspondence: kawater@fcritsumei.ac.jp

'Department of Biomedical Sciences, College of Life Sciences, Ritsumeikan University, Kusatsu, Japan

${ }^{2}$ Ritsumeikan Global Innovation Research Organization, Ritsumeikan University, Kusatsu, Japan
}

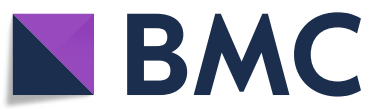

(c) The Author(s). 2020 Open Access This article is licensed under a Creative Commons Attribution 4.0 International License, which permits use, sharing, adaptation, distribution and reproduction in any medium or format, as long as you give

appropriate credit to the original author(s) and the source, provide a link to the Creative Commons licence, and indicate if changes were made. The images or other third party material in this article are included in the article's Creative Commons licence, unless indicated otherwise in a credit line to the material. If material is not included in the article's Creative Commons licence and your intended use is not permitted by statutory regulation or exceeds the permitted use, you will need to obtain permission directly from the copyright holder. To view a copy of this licence, visit http://creativecommons.org/licenses/by/4.0/ tically (self-renewal) and differentiate into any cell types except extraembryonic tissues (pluripotency), as observed in embryonic stem cells (ESCs) $[1,2]$. iPSCs currently hold promise for application in regenerative medicine, disease modeling, and drug screening [3, 4]. However, the risk of oncogenicity and low reprogramming efficiency interfere with the realization of the practical applications of iPSCs [4-6]. To solve the above mentioned problems, multiple approaches have been 
tested by modifying the combination of introduced reprogramming genes or using miRNAs and synthetic mRNAs, some of which result in $90-100 \%$ the reprogramming efficiency $[7,8]$. Nevertheless, it is critical to elucidate the molecular mechanisms underlying the reprogramming of somatic cells to iPSCs. During reprogramming, somatic cells undergo transitions in gene expression profile, epigenetic status, metabolic characteristics, and cellular morphology $[9,10]$. The importance of metabolic remodeling during the reprogramming to pluripotency has been proposed in many studies over the last decade [11-15]. The predominance of metabolism transits from oxidative phosphorylation (OXPHOS) to glycolysis has been observed during reprogramming. Glycolysis is a process with relatively low efficiency in terms of energy production; however, if the rate of metabolic flux is high enough, glycolysis could produce sufficient ATP for rapid cell proliferation. It is also important to note that glycolysis is essential for the biosynthesis of nucleic acids, amino acids, and lipids $[16,17]$. Interestingly, this aerobic glycolysis is similar to the metabolic property observed in cancer cells and other types of stem cells such as ESCs $[11,17]$. This metabolic character, known as the Warburg effect, is promoted by hypoxia-inducible factor 1 (HIF1), a core regulator of aerobic glycolysis [18]. HIF1 $\alpha$, the main subunit of HIF1, is rapidly degraded under normoxic conditions. However, under the hypoxic conditions found within a tumor tissue, HIF1 $\alpha$ is stabilized and regulates the transcription of its many target genes that contribute to tumor growth through increased cell proliferation and neovascularization [18]. HIF1 also promotes glycolysis by direct transcriptional regulation of glycolytic genes that switches the metabolic flux from OXPHOS to glycolysis. To date, several studies have investigated how the metabolic shift to glycolysis occurs in the reprogramming process, and they have demonstrated the importance of HIF1 in the metabolic shift. In this review, we summarize the current understanding of the dynamic changes in metabolic properties in the process of somatic cell reprogramming to iPSCs, with a special focus on the role of HIF1 in metabolic remodeling as a reprogramming enhancer.

\section{Metabolic features of PSCs}

The metabolic features of iPSCs are similar to those of ESCs, which are characterized by high glycolysis flux accompanied by low OXPHOS flux for ATP production as mentioned above [12, 19-23]. A similar metabolic phenomenon, called the Warburg effect, is observed in cancer cells [17, 24]. It is known that cancer metabolic processes are heterogeneous and vary depending on intratumoral oxygen concentrations. Particularly in hypoxic conditions, cancer cells generate ATP and metabolites for constructing cellular components through aerobic glycolysis regulated by a HIF1dependent mechanism [25-27], because intermediate metabolites in glycolysis and pentose phosphate pathways are required for high proliferative growth. Intermediate metabolites in glycolysis are also involved in stem cell properties. Several studies have revealed that glycolytic metabolism in ESCs is regulated by multiple mechanisms. For example, in the first step of glycolysis, hexokinase (HK) catalyzes phosphorylation of glucose to yield glucose-6-phosphate (G6P). Later, pyruvate kinase (PK) converts phosphoenolpyruvate to pyruvate. These glycolytic enzymes are expressed at a higher level in ESCs than in somatic cells. Increased activity of HK2, an isoform of $\mathrm{HK}$, and PKM2, an isoform of PK, maintains a high glycolytic rate in ESCs that contributes to pluripotency even in the absence of leukemia inhibitory factor (LIF) [28]. On the other hand, the inhibition of HK2 by 3-bromopyruvate causes a metabolic change from glycolysis to OXPHOS, leading to loss of pluripotency even in the presence of LIF $[15,28,29]$. Inactivation of pyruvate dehydrogenase (PDH) also maintains high glycolytic metabolism in ESCs [15]. In addition, core reprogramming factors play a critical role in sustaining high glycolytic flux. OCT4 is known to directly regulate HK2 and PKM2 transcription in ESCs [28]. Conditional double knockout of $c-M y c$ and $N-M y c$ in ESCs impairs self-renewal and pluripotency associated with downregulation of genes involved in cellular metabolism [30]. Conversely, inhibition of glycolysis using 2-deoxyglucose leads to the loss of pluripotency of PSCs [31]. Moreover, cell proliferation requires ATP and metabolic intermediates from the pentose phosphate pathway. In highly proliferative cancer cells, glycolysis provides G6P to the pentose phosphate pathway to generate ribose 5phophate for nucleotide biosynthesis [16, 32]. ESCs and iPSCs also utilize these metabolic intermediates for rapid cell proliferation and pluripotency [12, 15, 21, 29]. Several studies have demonstrated that upregulation of genes involved in glycolysis and pentose phosphate pathways results in epigenetic changes in the early stage of iPSC reprogramming [12, 15, 22].

Despite the relatively low contribution of OXPHOS to ATP production in PSCs compared to that in somatic cells, mitochondria still play an important role in biosynthesis of metabolic intermediates [33]. Aerobic glycolysis in cancer cells is similar to that in PSCs, but not exactly same. In glycolysis, cancer cells use pyruvate to generate lactate, whereas in PSCs, glucose is increasingly converted to acetyl-coenzyme A (acetyl-CoA) [33]. Subsequently, acetyl-CoA is converted to citrate by citrate synthase in the mitochondria. Citrate is then exported from the mitochondria to the cytoplasm and is again converted to cytosolic acetyl-CoA by ATP-citrate lyase. 
Cytosolic acetyl-CoA acetylates histones in ESCs to maintain the open state of the chromatin structure, leading to pluripotency maintenance in PSCs [33, 34]. In contrast, the loss of acetyl-CoA results in histone deacetylation and loss of pluripotency in PSCs [33]. Acetyl$\mathrm{CoA}$, together with glycine produced in the threonine metabolism pathway, is also essential for Sadenosylmethionine (SAM) synthesis [35]. SAM contributes to histone methylation, for example, H3K4me3, leading to the pluripotency of "naïve" mouse ESCs. In addition, human "primed" PSCs utilize methionine to generate SAM and maintain H3K4me3 levels for the maintenance of the pluripotency [36]. In terms of the amino acid utility, human primed PSCs also rely on glutamine oxidation to synthesize ATP [37]. Even in the absence of the glycolytic flux, primed PSCs can survive through nucleotides and glutathione synthesis and energy production via the glutamine metabolic pathway [37]. In contrast, glutamine depletion results in a decrease in $\alpha \mathrm{KG}$ levels leading to histone methylation and subsequent differentiation [38]. However, in naïve PSCs, the absence of glutamine does not affect cell proliferation and histone methylation when glucose is available as an energy source [38]. This might be because the origin of naïve and primed PSCs is different. The former is established from the inner cell mass of the blastocyst, while the latter is derived from the postimplantation epiblast where there is no blood supply in utero $[39,40]$.

The mitochondrial electron transport chain (ETC) is fully functional in ESCs. Uncoupling protein 2 (UCP2) in ESCs shifts from OXPHOS to glycolysis by shunting pyruvate out of mitochondria [41]. UCP2 uncouples glucose oxidation from ATP production in ETC, thereby reducing reactive oxygen species (ROS) generation. As excessive ROS have the potential to damage nucleic acids, lipids, and proteins in cells, ROS production needs to be maintained at a low level possibly by UCP2 for stem cell maintenance [22, 42]. Therefore, mitochondrion is a critical subcellular organelle that maintains PSC properties through UCP2-dependent modulation of OXPHOS flux for ROS reduction as well as increasing the biosynthesis of metabolic intermediates for epigenetic modification. In addition, HIF1 is involved in the transcriptional regulation of several glycolytic genes that enhance the metabolic flux of glycolysis. Figure 1 shows a schematic illustration of glycolytic metabolism and the involvement of HIF1.

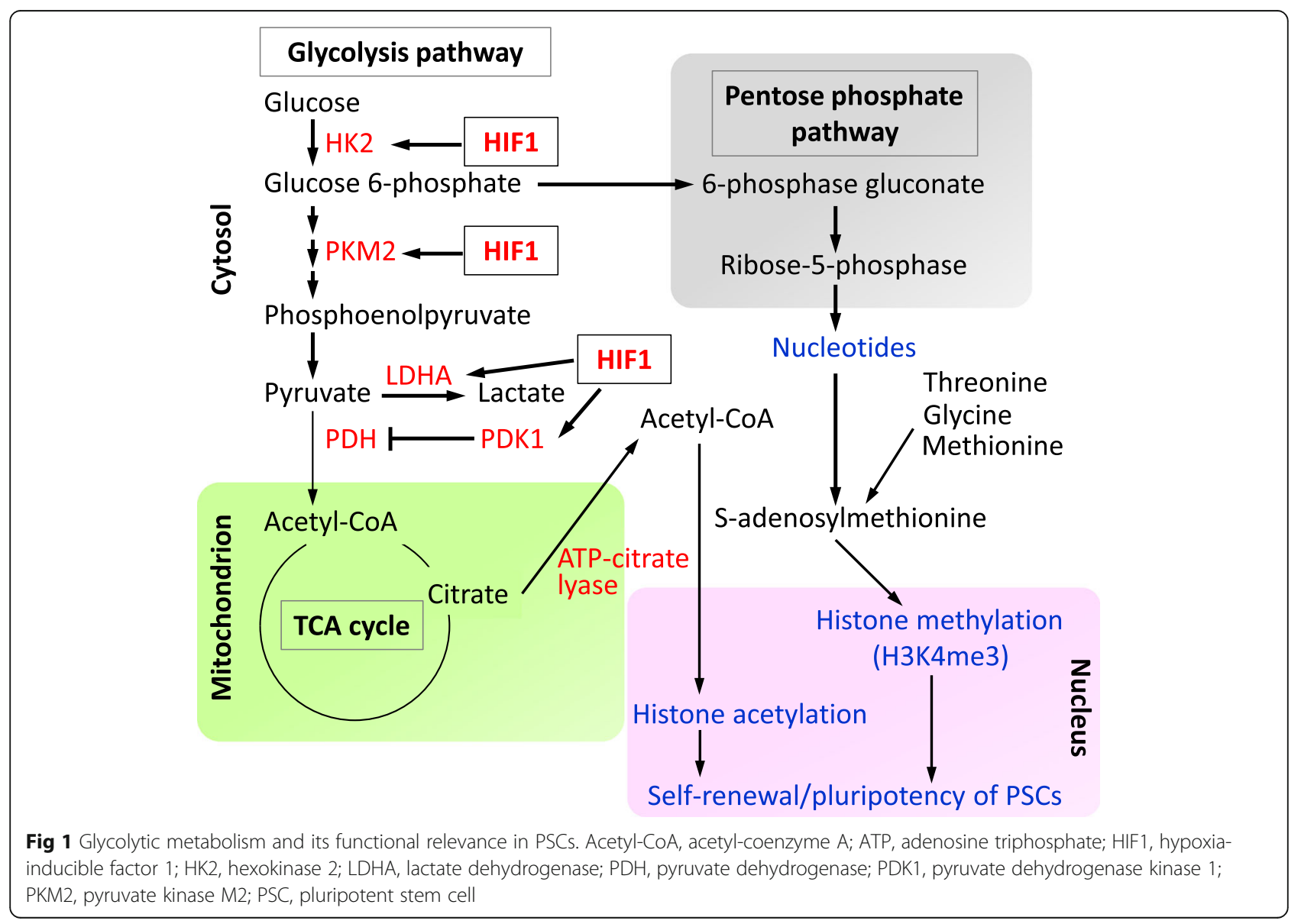


Metabolic shift during reprogramming to iPSCs

As described, iPSCs predominantly utilize glycolysis to generate ATP and intermediates that contribute to pluripotency and rapid cell proliferation. A metabolic shift from OXPHOS to glycolysis occurs in somatic cells undergoing reprogramming into iPSCs [14, 15, 22]. Several recent studies have investigated the mechanism underlying the metabolic shift during the reprogramming. Glycolytic genes are upregulated early before the induction of pluripotency genes, and they remain upregulated during reprogramming [12, 13, 43, 44]. Glucose uptake and lactate production are constantly increased by upregulation of the glucose transporter GLUT1 and lactate dehydrogenase A (LDHA), which is consistent with the gradual metabolic shift from OXPHOS to glycolysis [12]. Moreover, activation of glycolysis results in improved reprogramming efficiency, whereas inhibition of glycolysis reduces reprogramming efficiency [12, 14, 15, 22].

Pyruvate dehydrogenase kinase 1 (PDK1) is one of metabolic enzymes involved in aerobic glycolysis activation. PDK1 phosphorylates and inactivates the PDH complex, which catalyzes pyruvate into acetyl-CoA [45].
As the PDK1 protein is more stabilized in PSCs than in differentiated somatic cells, possibly via a HIFdependent regulation, reprogramming cells may show enhanced glycolytic activity [15] (Fig. 1). In addition, AKT activity is correlated with the upregulation of glycolytic genes and increases lactate production, resulting in improvement of the reprogramming efficiency $[46,47]$. TCL1, a protooncogene that phosphorylates AKT as a co-activator, is upregulated by KLF4 through direct transcriptional regulation. This increased expression of TCL1 was observed in late-stage reprogramming [48, 49]. TCL1 also reduces OXPHOS flux by inhibiting mitochondrial polynucleotide phosphorylase. TCL1 thus promotes metabolic shift in an AKT-dependent manner that increases the reprogramming efficiency $[48,50]$ (Fig. 2).

Despite the importance of glycolysis in reprogramming to iPSCs, Kida et al. revealed that OXPHOS is transiently increased at the early stage of reprogramming, at which mitochondrial proteins are upregulated [51]. This OXPHOS burst leads to the metabolic shift and is ultimately required for the establishment of induced

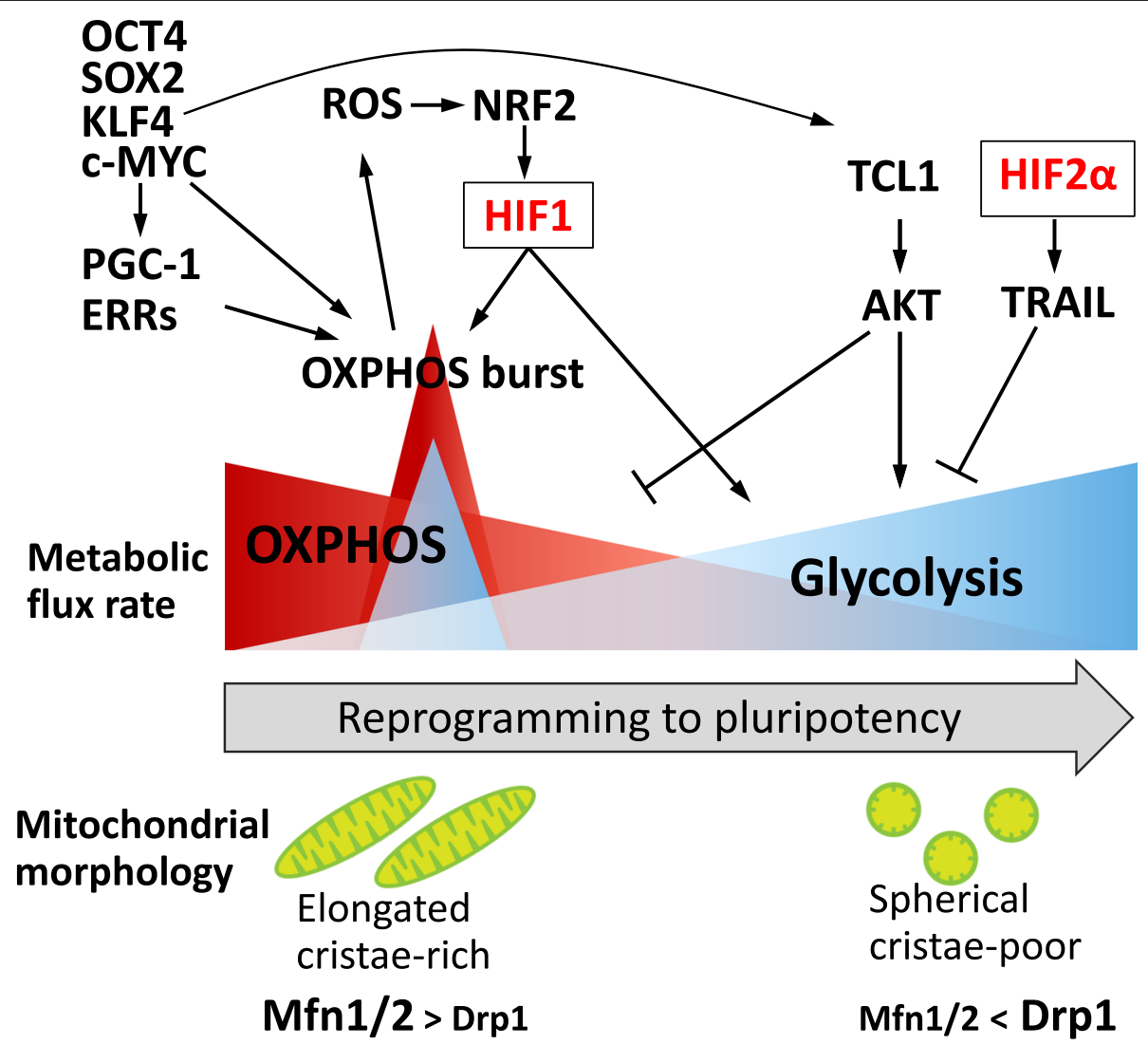

Fig 2 Metabolic shift and its related molecules during reprogramming to pluripotency. DRP1, dynamin-related protein 1; ERRs, estrogen-related nuclear receptors; HIF, hypoxia-inducible factor; Mfn, mitofusin; NRF2, nuclear factor erythroid 2-related factor 2; OXPHOS, oxidative phosphorylation; PGC-1, peroxisome proliferator-activated receptor gamma coactivator-1; ROS, reactive oxygen species; TRAIL, TNF-related apoptosis-inducing ligand 
pluripotency. Mechanistically, estrogen-related nuclear receptors (ERR $\alpha$ and ERR $\gamma$ ) and their co-activators, PGC- $1 \alpha$ and PGC- $1 \beta$, are upregulated transiently during early-stage reprogramming triggered by the introduction of reprogramming factors, particularly c-MYC. PGC-1/ ERRs subsequently induce the hyper-energetic state of metabolism necessary for iPSC production. In contrast, the inhibition of ERR expression fails to induce successful reprogramming [51]. Moreover, the fact that c-MYC can significantly upregulate the expression levels of glycolysis- and OXPHOS-related enzymes demonstrates the deep involvement of c-MYC in metabolic shift at the early stage of reprogramming [52]. Considering that cMYC, together with HIF1, regulates metabolic remodeling in cancer cells under hypoxic conditions [53, 54], cMYC and HIF1 may cooperate to induce metabolic shift during iPSC reprogramming as well. Hawkins et al. similarly reported that OXPHOS burst during early reprogramming is regulated by nuclear factor erythroid 2related factor 2 (NRF2) via the induction of HIF1mediated glycolytic shift and glucose redistribution to the pentose phosphate pathway [55]. In contrast, inhibition of NRF2 by an E3 ubiquitin ligase adaptor, KEAP1, overexpression, or knockdown of NRF2, reduces the reprogramming efficiency [55, 56]. Moreover, prior to NRF2 upregulation, ROS production was increased presumably as a result of elevated mitochondrial activity associated with OXPHOS burst. As NRF2 is known as a master regulator of the antioxidant response, it may protect cells from oxidative stress during reprogramming [55]. Although it remains unclear how the transient hyperenergetic state is decreased to the proper level, OXPHOS burst is essential to initiate and ensures the progress of reprogramming to pluripotency.

Mitochondrial morphology differs significantly between somatic cells and iPSCs. Introduction of the reprogramming factors induces regression from mature tubular and cristae-rich mitochondria to immature spherical and cristae-poor mitochondria. Mitochondrial mass is also decreased by iPSC reprogramming [12]. Forced expression of c-MYC affects mitochondrial morphology, which changes from tubular to fragmented, accompanied by upregulation of the mitochondrial fission regulator, dynamin-related protein 1 (DRP1), and elevation of the mitochondrial membrane potential as a mitochondrial function indicator [52]. As for the effect of modulation of mitochondrial dynamics, DRP1 inhibition reduces reprogramming efficiency $[57,58]$. In contrast, deficiency of mitofusin 1 and 2 , the mitochondrial fusion regulators, facilitates metabolic conversion to glycolysis and promotes iPSC production [59]. Thus, mitochondria remodeling occurs during reprogramming and influences the efficacy of iPSC production $[22,23,60]$. As discussed so far, these dynamic changes in mitochondrial structure during reprogramming may be highly correlated to mitochondrial function such as OXPHOS and ETC activity, linking to metabolic shift that promotes the acquisition of pluripotency. Figure 2 illustrates the metabolic shift and its associated regulatory molecules during reprogramming to pluripotency.

\section{HIF1 for metabolic reprogramming}

As mentioned above, PSCs predominantly utilize glycolysis for ATP generation, akin to the cancer cell metabolism. Cancer cells utilize glycolysis for ATP production regardless of oxygen conditions; this aerobic glycolysis is known as the Warburg effect $[17,24]$. It is known that the primary regulator of the Warburg effect is HIF1 [18, 61]. HIF1 regulates gene transcription of over 100 genes controlling cell metabolism, survival, motility, angiogenesis, hematopoiesis, and other cellular functions in response to hypoxia. HIF1 functions as a heterodimer comprising HIF1 $\alpha$ and HIF1 $\beta$ subunits, and the stability of the HIF1 $\alpha$ protein depends on oxygen concentration. Under normoxic conditions, proline residues of HIF1 $\alpha$ are hydroxylated by prolyl hydroxylase (PHD). Hydroxyprolines allow the binding HIF1 $\alpha$ with the von Hippel-Lindau protein, resulting in ubiquitination by an E3 ubiquitin ligase and subsequent degradation by the proteasome [62]. Under hypoxic condition, HIF1 $\alpha$ escapes from hydroxylation by PHD because PHD activity is repressed by insufficient oxygen concentrations. HIF1 $\alpha$ then accumulates and dimerizes with HIF1 $\beta$. The dimerized HIF1 then drives the transcription of hypoxia-responsive genes (Fig. 3). Factor inhibiting HIF1 (FIH1), similar to the PHD family members, is a $\mathrm{Fe}^{2+}$ - and 2-oxoglutrate-dependent dioxygenase. In normoxia, FIH1 hydroxylates an asparagine residue of HIF $1 \alpha$ that prevents the transcription co-activators p300 and CBP recruitment. Although FIH1 is less sensitive to hypoxia than PHD, hypoxia inactivates FIH1, allowing further tuning of the transcriptional activity of HIF1 by p300/CBP (Fig. 3).

It was previously reported that hypoxia promotes iPSC generation [63], and an HIF family member, HIF2 $\alpha$, which is also hypoxia-activated and dimerizes with HIF1 $\beta$, improves the reprogramming efficiency by suppressing the tumor suppressor p53 [64]. More recent reports have demonstrated that HIF family plays a critical role in the metabolic shift during reprogramming. HIF1 $\alpha$ increases the expression of glycolytic genes and promotes glycolysis during reprogramming, whereas the knockdown of HIF $1 \alpha$ and HIF $2 \alpha$ negatively regulates reprogramming $[13,43]$. Mathieu et al. showed that HIF1 $\alpha$ and HIF $2 \alpha$ are stabilized under normoxic conditions during reprogramming, and both HIF $1 \alpha$ and HIF $2 \alpha$ are required to initiate the metabolic shift at the early stage of reprogramming. However, prolonged HIF2 $\alpha$ 


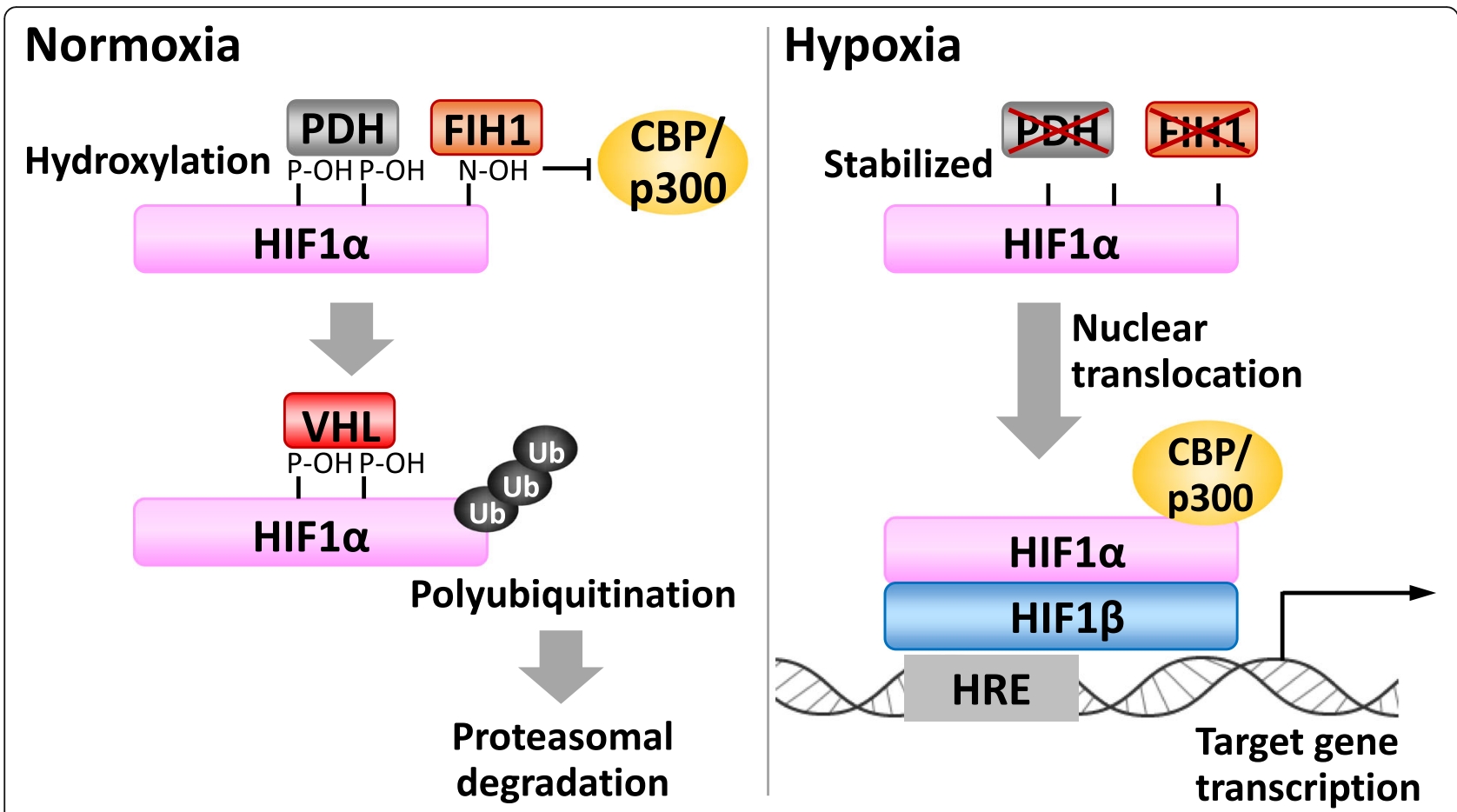

Fig 3 HIF1a turnover and its transcription function. FIH1, factor inhibiting HIF1; HIF, hypoxia-inducible factor; HRE, HIF-responsive element; PHD, prolyl hydroxylase; VHL, von Hippel-Lindau protein

activation represses iPSC formation, while prolonged HIF $1 \alpha$ activation increases the reprogramming efficiency [13]. HIF $2 \alpha$ activation at the late stage of reprogramming may repress reprogramming through TNF-related apoptosis-inducing ligand (TRAIL). TRAIL inhibits iPSC formation by repressing apoptotic caspase 3 activity, and inhibition of TRAIL activity enhances iPSC generation [13] (Fig. 2). Therefore, it is suggested that HIF1 $\alpha$ and HIF $2 \alpha$ have stage-dependent distinctive roles in promoting iPSC reprogramming. Furthermore, Prigione et al. demonstrated that HIF1 $\alpha$ promotes the reprogramming efficiency through upregulation of metabolic enzymes PK and PDK, promoting the metabolic shift to glycolysis [43]. They also confirmed that the depletion of HIF1 $\alpha$ significantly decreased iPSC formation. Mechanistically, the expression of PKM2, PDK1, and PDK3 is upregulated by HIF1 activation during reprogramming, while the knockdown of HIF1 $\alpha$ prevents an increase in the expression levels of these glycolytic enzymes. Moreover, the introduction of reprogramming factors upregulates the expression of PDK1 [43]. These studies thus indicate that HIF $1 \alpha$ and HIF $2 \alpha$ play a pivotal role in the metabolic shift to glycolysis at the early stage, and that HIF $1 \alpha$ maintains a high glycolysis flux in the later phase of reprogramming to pluripotency.

Gene introduction of the reprogramming factors elevates HIF1 activity that promotes iPSC production. Taking the above into consideration, pharmacological stabilization of HIF1 $\alpha$ may enable to promote the successful reprogramming even in normoxic conditions. In this context, there are PHD inhibitors known as HIF $1 \alpha$ stabilizers that are commercially available for the treatment of renal anemia through promoting the biosynthesis of erythropoietin, one of the common HIF1 target genes, encoding a hormone that stimulates blood production [65]. Thus, although a HIF1 $\alpha$ stabilizer may have the potential to trigger tumorigenicity via HIF1mediated mechanisms [18], it might be of importance to utilize HIF1 $\alpha$ stabilizers for efficient production of iPSCs.

\section{Conclusion}

iPSCs are a promising cell source for regenerative medicine, disease modeling, and drug discovery. However, the molecular basis of the iPSC reprogramming process remains not fully uncovered. Recent studies have highlighted the importance of the metabolism of iPSCs, characterized by glycolysis activation, compared to that of its somatic counterparts. Glycolytic metabolism provides ATP and drives the pentose phosphate pathway, contributing to rapid cell proliferation. Despite their lower contribution to OXPHOS in iPSCs than in somatic cells, mitochondria in iPSCs are also highly involved in the acquisition and maintenance of induced pluripotency by providing metabolic intermediates required for epigenetic modifications as well as by reducing ROS 
production. In the early stage of reprogramming, a transient increase in the OXPHOS rate is an essential event. This OXPHOS burst then declines that reduces the ROS production, followed by sustained glycolysis activation. We also introduced HIF1 $\alpha$, which activates the transcription of many target genes to promote the glycolysis-shifted metabolism necessary for successful reprogramming. In this context, activation of HIF1 by pharmacological stabilizers could be useful for efficient production of iPSCs. Further investigations of the interplaying networks of transcriptional, epigenetic, and metabolic properties in iPSC reprogramming will lead to significant progress in understanding the detailed mechanisms of cell plasticity.

\section{Abbreviations \\ Acetyl-CoA: Acetyl-coenzyme A; ATP: Adenosine triphosphate; DRP1: Dynamin-related protein 1; ERR: Estrogen-related nuclear receptor; ESC: Embryonic stem cell; ETC: Electron transport chain; FIH1: Factor inhibiting HIF-1; G6P: Glucose-6-phosphate; GLUT4: Glucose transporter 4; HIF: Hypoxia-inducible factor; HK: Hexokinase; iPSC: Induced pluripotent stem cell; LDHA: Lactate dehydrogenase A; LIF: Leukemia inhibitory factor; NRF2: Nuclear factor erythroid-related 2 factor 2; OXPHOS: Oxidative phosphorylation; PDH: Pyruvate dehydrogenase; PDK: Pyruvate dehydrogenase kinase; PGC-1: Peroxisome proliferator-activated receptor gamma coactivator-1; PHD: Prolyl hydroxylase; PK: Pyruvate kinase; ROS: Reactive oxygen species; SAM: S-adenosylmethionine; TRAIL: TNF- related apoptosis-inducing ligand; UCP2: Uncoupling protein 2}

\section{Acknowledgements}

Not applicable

\section{Authors' contributions}

Conceptualization, T.K.; writing—original draft preparation, T.I., S.N., and T.K. writing-review and editing, T. I., S.N., T.U., Y.H., and T.K.; visualization, S.N. and T.K.; supervision, S.N. and T.K.; funding acquisition, S.N. and T.K. All authors have read and agreed to the published version of the manuscript.

\section{Funding}

This article was funded by JSPS KAKEN (16H04725 and 18K15017) and Inamori Foundation Research Grant for Natural Sciences.

\section{Availability of data and materials}

Not applicable

\section{Ethics approval and consent to participate}

Not applicable

\section{Consent for publication}

Not applicable

\section{Competing interests}

There is no competing interest to declare.

Received: 28 February 2020 Accepted: 22 April 2020

Published online: 12 May 2020

\section{References}

1. Takahashi K, Tanabe K, Ohnuki M, Narita M, Ichisaka T, Tomoda K, Yamanaka S. Induction of pluripotent stem cells from adult human fibroblasts by defined factors. Cell. 2007:131:861-72.

2. Takahashi K, Yamanaka S. Induction of pluripotent stem cells from mouse embryonic and adult fibroblast cultures by defined factors. Cell. 2006;126: 663-76.

3. Kim C, Wong J, Wen JY, Wang SR, Wang C, Spiering S, Kan NG, Forcales S, Puri PL, Leone TC, Marine JE, Calkins H, Kelly DP, Judge DPChen HSV.
Studying arrhythmogenic right ventricular dysplasia with patient-specific iPSCs. Nature. 2013:494:105-110.

4. Takahashi K, Yamanaka S. A decade of transcription factor-mediated reprogramming to pluripotency. Nat Rev Mol Cell Biol. 2016;17:183-93.

5. Kawamura T, Suzuki J, Wang W, Menendez S, Morera LB, Raya A, Wahl GM, Izpisua Belmonte JC. Linking the p53 tumour suppressor pathway to somatic cell reprogramming. Nature. 2009;460:1140-4.

6. Yamanaka S. Elite and stochastic models for induced pluripotent stem cell generation. Nature. 2009;460:49-52.

7. Kogut I, McCarthy SM, Pavlova M, Astling DP, Chen X, Jakimenko A, Jones KL, Getahun A, Cambier JC, Pasmooij AMG, Jonkman MF, Roop DRBilousova G. High-efficiency RNA-based reprogramming of human primary fibroblasts. Nat Commun. 2018:9:745.

8. Rais Y, Zviran A, Geula S, Gafni O, Chomsky E, Viukov S, Mansour AA, Caspi I, Krupalnik V, Zerbib M, Maza I, Mor N, Baran D, Weinberger L, Jaitin DA, LaraAstiaso D, Blecher-Gonen R, Shipony Z, Mukamel Z, Hagai T, Gilad S, Amann-Zalcenstein D, Tanay A, Amit I, Novershtern NHanna JH. Deterministic direct reprogramming of somatic cells to pluripotency. Nature. 2013;502:65-70.

9. Boyer LA, Lee TI, Cole MF, Johnstone SE, Levine SS, Zucker JP, Guenther MG, Kumar RM, Murray HL, Jenner RG, Gifford DK, Melton DA, Jaenisch RYoung RA. Core transcriptional regulatory circuitry in human embryonic stem cells. Cell. 2005;122:947-56.

10. Orkin SH, Hochedlinger K. Chromatin connections to pluripotency and cellular reprogramming. Cell. 2011;145:835-50.

11. Folmes CD, Dzeja PP, Nelson TJ, Terzic A. Metabolic plasticity in stem cell homeostasis and differentiation. Cell Stem Cell. 2012;11:596-606.

12. Folmes CD, Nelson TJ, Martinez-Fernandez A, Arrell DK, Lindor JZ, Dzeja PP, Ikeda Y, Perez-Terzic C, Terzic A. Somatic oxidative bioenergetics transitions into pluripotency-dependent glycolysis to facilitate nuclear reprogramming. Cell Metab. 2011;14:264-71.

13. Mathieu J, Zhou W, Xing Y, Sperber H, Ferreccio A, Agoston Z, Kuppusamy KT, Moon RT, Ruohola-Baker H. Hypoxia-inducible factors have distinct and stage-specific roles during reprogramming of human cells to pluripotency. Cell Stem Cell. 2014;14:592-605.

14. Panopoulos AD, Yanes O, Ruiz S, Kida YS, Diep D, Tautenhahn R, Herrerias A, Batchelder EM, Plongthongkum N, Lutz M, Berggren WT, Zhang K, Evans RM, Siuzdak Glzpisua Belmonte JC. The metabolome of induced pluripotent stem cells reveals metabolic changes occurring in somatic cell reprogramming. Cell Res. 2012;22:168-77.

15. Varum S, Rodrigues AS, Moura MB, Momcilovic O, Easley CA, RamalhoSantos J, Van Houten BSchatten G. Energy metabolism in human pluripotent stem cells and their differentiated counterparts. PLoS One. 2011; 6:e20914.

16. DeBerardinis RJ, Lum JJ, Hatzivassiliou G, Thompson CB. The biology of cancer: metabolic reprogramming fuels cell growth and proliferation. Cell Metab. 2008:7:11-20.

17. Vander Heiden MG, Cantley LC, Thompson CB. Understanding the Warburg effect: the metabolic requirements of cell proliferation. Science. 2009;324: 1029-33.

18. Semenza GL. Defining the role of hypoxia-inducible factor 1 in cancer biology and therapeutics. Oncogene. 2010;29:625-34.

19. Cho YM, Kwon S, Pak YK, Seol HW, Choi YM, Park DJ, Park KS, Lee HK. Dynamic changes in mitochondrial biogenesis and antioxidant enzymes during the spontaneous differentiation of human embryonic stem cells. Biochem Biophys Res Commun. 2006;348:1472-8.

20. Chung S, Arrell DK, Faustino RS, Terzic ADzeja PP. Glycolytic network restructuring integral to the energetics of embryonic stem cell cardiac differentiation. J Mol Cell Cardiol. 2010;48:725-34.

21. Kondoh $\mathrm{H}$, Lleonart ME, Nakashima Y, Yokode M, Tanaka M, Bernard D, Gil JBeach D. A high glycolytic flux supports the proliferative potential of murine embryonic stem cells. Antioxid Redox Signal. 2007;9:293-9.

22. Prigione A, Fauler B, Lurz R, Lehrach HAdjaye J. The senescence-related mitochondrial/oxidative stress pathway is repressed in human induced pluripotent stem cells. Stem Cells. 2010;28:721-33.

23. Suhr ST, Chang EA, Tjong J, Alcasid N, Perkins GA, Goissis MD, Ellisman MH, Perez GI, Cibelli JB. Mitochondrial rejuvenation after induced pluripotency. PLoS One. 2010:5:e14095.

24. Warburg O. On the origin of cancer cells. Science. 1956;123:309-14.

25. Nakazawa MS, Keith B, Simon MC. Oxygen availability and metabolic adaptations. Nat Rev Cancer. 2016;16:663-73. 
26. Gatenby RA, Gillies RJ. Why do cancers have high aerobic glycolysis? Nat Rev Cancer. 2004;4:891-9.

27. Xiao Z, Dai Z, Locasale JW. Metabolic landscape of the tumor microenvironment at single cell resolution. Nat Commun. 2019;10:3763.

28. Kim H, Jang H, Kim TW, Kang BH, Lee SE, Jeon YK, Chung DH, Choi J, Shin J, Cho EJ, Youn HD. Core pluripotency factors directly regulate metabolism in embryonic stem cell to maintain pluripotency. Stem Cells. 2015;33:2699-711.

29. Rodrigues AS, Pereira SL, Correia M, Gomes A, Perestrelo T, Ramalho-Santos J. Differentiate or die: 3-bromopyruvate and pluripotency in mouse embryonic stem cells. PLoS One. 2015;10:e0135617.

30. Varlakhanova NV, Cotterman RF, de Vries WN, Morgan J, Donahue LR, Murray S, Knowles BB, Knoepfler PS. myc maintains embryonic stem cell pluripotency and self-renewal. Differentiation. 2010;80:9-19.

31. Takashima Y, Guo G, Loos R, Nichols J, Ficz G, Krueger F, Oxley D, Santos F, Clarke J, Mansfield W, Reik W, Bertone P, Smith A. Resetting transcription factor control circuitry toward ground-state pluripotency in human. Cell. 2014;158:1254-69.

32. Locasale JW, Cantley LC. Metabolic flux and the regulation of mammalian cell growth. Cell Metab. 2011;14:443-51.

33. Moussaieff A, Rouleau M, Kitsberg D, Cohen M, Levy G, Barasch D, Nemirovski A, Shen-Orr S, Laevsky I, Amit M, Bomze D, Elena-Herrmann B, Scherf T, Nissim-Rafinia M, Kempa S, Itskovitz-Eldor J, Meshorer E, Aberdam D, Nahmias Y. Glycolysis-mediated changes in acetyl-CoA and histone acetylation control the early differentiation of embryonic stem cells. Cell Metab. 2015;21:392-402

34. Wellen KE, Hatzivassiliou G, Sachdeva UM, Bui TV, Cross JR, Thompson CB. ATP-citrate lyase links cellular metabolism to histone acetylation. Science. 2009:324:1076-80

35. Shyh-Chang N, Locasale JW, Lyssiotis CA, Zheng Y, Teo RY, Ratanasirintrawoot S, Zhang J, Onder T, Unternaehrer JJ, Zhu H, Asara JM, Daley GQ, Cantley LC. Influence of threonine metabolism on Sadenosylmethionine and histone methylation. Science. 2013;339:222-6.

36. Shiraki N, Shiraki Y, Tsuyama T, Obata F, Miura M, Nagae G, Aburatani H, Kume K, Endo F, Kume S. Methionine metabolism regulates maintenance and differentiation of human pluripotent stem cells. Cell Metab. 2014;19: 780-94.

37. Tohyama S, Fujita J, Hishiki T, Matsuura T, Hattori F, Ohno R, Kanazawa H, Seki T, Nakajima K, Kishino Y, Okada M, Hirano A, Kuroda T, Yasuda S, Sato Y, Yuasa S, Sano M, Suematsu M, Fukuda K. Glutamine oxidation is indispensable for survival of human pluripotent stem cells. Cell Metab. 2016: 23:663-74.

38. Carey BW, Finley LW, Cross JR, Allis CD, Thompson CB. Intracellular alphaketoglutarate maintains the pluripotency of embryonic stem cells. Nature. 2015;518:413-6

39. Gu W, Gaeta X, Sahakyan A, Chan AB, Hong CS, Kim R, Braas D, Plath K, Lowry WE, Christofk HR. Glycolytic metabolism plays a functional role in regulating human pluripotent stem cell state. Cell Stem Cell. 2016;19:476-90.

40. Zhou W, Choi M, Margineantu D, Margaretha L, Hesson J, Cavanaugh C, Blau CA, Horwitz MS, Hockenbery D, Ware C, Ruohola-Baker H. HIF1alpha induced switch from bivalent to exclusively glycolytic metabolism during ESC-to-EpiSC/hESC transition. EMBO J. 2012;31:2103-16.

41. Zhang J, Khvorostov I, Hong JS, Oktay Y, Vergnes L, Nuebel E, Wahjudi PN, Setoguchi K, Wang G, Do A, Jung HJ, McCaffery JM, Kurland IJ, Reue K, Lee WN, Koehler CM, Teitell MA. UCP2 regulates energy metabolism and differentiation potential of human pluripotent stem cells. EMBO J. 2011;30: 4860-73.

42. Armstrong L, Tilgner K, Saretzki G, Atkinson SP, Stojkovic M, Moreno R, Przyborski S, Lako M. Human induced pluripotent stem cell lines show stress defense mechanisms and mitochondrial regulation similar to those of human embryonic stem cells. Stem Cells. 2010;28:661-73.

43. Prigione A, Rohwer N, Hoffmann S, Mlody B, Drews K, Bukowiecki R, Blumlein K, Wanker EE, Ralser M, Cramer T, Adjaye J. HIF1alpha modulates cell fate reprogramming through early glycolytic shift and upregulation of PDK1-3 and PKM2. Stem Cells. 2014;32:364-76.

44. Hansson J, Rafiee MR, Reiland S, Polo JM, Gehring J, Okawa S, Huber W, Hochedlinger K, Krijgsveld J. Highly coordinated proteome dynamics during reprogramming of somatic cells to pluripotency. Cell Rep. 2012;2:1579-92.

45. Gudi R, Bowker-Kinley MM, Kedishvili NY, Zhao Y, Popov KM. Diversity of the pyruvate dehydrogenase kinase gene family in humans. J Biol Chem. 1995; 270:28989-94.
46. Elstrom RL, Bauer DE, Buzzai M, Karnauskas R, Harris MH, Plas DR, Zhuang H, Cinalli RM, Alavi A, Rudin CM, Thompson CB. Akt stimulates aerobic glycolysis in cancer cells. Cancer Res. 2004;64:3892-9.

47. Yu Y, Liang D, Tian Q, Chen X, Jiang B, Chou BK, Hu P, Cheng L, Gao P, Li JWang G. Stimulation of somatic cell reprogramming by ERas-Akt-FoxO1 signaling axis. Stem Cells. 2014;32:349-63.

48. Nishimura K, Aizawa S, Nugroho FL, Shiomitsu E, Tran YTH, Bui PL, Borisova E, Sakuragi Y, Takada H, Kurisaki A, Hayashi Y, Fukuda A, Nakanishi M, Hisatake K. A role for KLF4 in promoting the metabolic shift via TCL1 during induced pluripotent stem cell generation. Stem Cell Reports. 2017;8:787-801.

49. Laine J, Kunstle G, Obata T, Sha M, Noguchi M. The protooncogene TCL1 is an Akt kinase coactivator. Mol Cell. 2000;6:395-407.

50. Khaw SL, Min-Wen C, Koh CG, Lim B, Shyh-Chang N. Oocyte factors suppress mitochondrial polynucleotide phosphorylase to remodel the metabolome and enhance reprogramming. Cell Rep. 2015;12:1080-8.

51. Kida YS, Kawamura T, Wei Z, Sogo T, Jacinto S, Shigeno A, Kushige H, Yoshihara E, Liddle C, Ecker JR, Yu RT, Atkins AR, Downes M, Evans RM. ERRs mediate a metabolic switch required for somatic cell reprogramming to pluripotency. Cell Stem Cell. 2015;16:547-55.

52. Prieto J, Seo AY, Leon M, Santacatterina F, Torresano L, Palomino-Schatzlein M, Gimenez K, Vallet-Sanchez A, Ponsoda X, Pineda-Lucena A, Cuezva JM, Lippincott-Schwartz J, Torres J. MYC induces a hybrid energetics program early in cell reprogramming. Stem Cell Reports. 2018;11:1479-92.

53. Gordan JD, Thompson CB, Simon MC. HIF and c-Myc: sibling rivals for control of cancer cell metabolism and proliferation. Cancer cell. 2007;12: 108-13.

54. Huang LE. Carrot and stick: HIF-alpha engages c-Myc in hypoxic adaptation. Cell Death Differ. 2008:15:672-7.

55. Hawkins KE, Joy S, Delhove JM, Kotiadis VN, Fernandez E, Fitzpatrick LM, Whiteford JR, King PJ, Bolanos JP, Duchen MR, Waddington SN, TR MK NRF2 orchestrates the metabolic Shift during induced pluripotent stem cell reprogramming. Cell Rep. 2016;14:1883-91.

56. Jang J, Wang Y, Kim HS, Lalli MA, Kosik KS. Nrf2, a regulator of the proteasome, controls self-renewal and pluripotency in human embryonic stem cells. Stem Cells. 2014;32:2616-25.

57. Prieto J, Leon M, Ponsoda X, Sendra R, Bort R, Ferrer-Lorente R, Raya A, Lopez-Garcia C, Torres J. Early ERK1/2 activation promotes DRP1-dependent mitochondrial fission necessary for cell reprogramming. Nat Commun. 2016; 7:11124.

58. Vazquez-Martin A, Cufi S, Corominas-Faja B, Oliveras-Ferraros C, Vellon L, Menendez JA. Mitochondrial fusion by pharmacological manipulation impedes somatic cell reprogramming to pluripotency: new insight into the role of mitophagy in cell stemness. Aging (Albany NY). 2012;4:393-401.

59. Son MJ, Kwon Y, Son MY, Seol B, Choi HS, Ryu SW, Choi C, Cho YS. Mitofusins deficiency elicits mitochondrial metabolic reprogramming to pluripotency. Cell Death Differ. 2015;22:1957-69.

60. Bukowiecki R, Adjaye J, Prigione A. mitochondrial function in pluripotent stem cells and cellular reprogramming. Gerontology. 2014;60:174-82.

61. Lu H, Forbes RA, Verma A. Hypoxia-inducible factor 1 activation by aerobic glycolysis implicates the Warburg effect in carcinogenesis. J Biol Chem. 2002;277:23111-5.

62. Kaelin WG Jr, Ratcliffe PJ. Oxygen sensing by metazoans: the central role of the HIF hydroxylase pathway. Mol Cell. 2008;30:393-402.

63. Yoshida Y, Takahashi K, Okita K, Ichisaka T, Yamanaka S. Hypoxia enhances the generation of induced pluripotent stem cells. Cell Stem Cell. 2009;5: 237-41.

64. Das B, Bayat-Mokhtari R, Tsui M, Lotfi S, Tsuchida R, Felsher DW, Yeger H. HIF-2alpha suppresses p53 to enhance the stemness and regenerative potential of human embryonic stem cells. Stem Cells. 2012;30:1685-95.

65. Maxwell PH, Eckardt KU. HIF prolyl hydroxylase inhibitors for the treatment of renal anaemia and beyond. Nat Rev Nephrol. 2016;12:157-68.

\section{Publisher's Note}

Springer Nature remains neutral with regard to jurisdictional claims in published maps and institutional affiliations. 